\title{
Genomic mapping of the MHC transactivator CIITA using an integrated ChIP-seq and genetical genomics approach
}

\author{
Daniel Wong ${ }^{1 \dagger}$, Wanseon Lee ${ }^{1 \dagger}$, Peter Humburg ${ }^{1}$, Seiko Makino ${ }^{1}$, Evelyn Lau' ${ }^{1}$ Vivek Naranbhai ${ }^{1}$, \\ Benjamin P Fairfax', Kenneth Chan ${ }^{2}$, Katharine Plant ${ }^{1}$ and Julian C Knight ${ }^{\text {* }}$
}

\begin{abstract}
Background: The master transactivator CIITA is essential to the regulation of Major Histocompatibility Complex (MHC) class II genes and an effective immune response. CIITA is known to modulate a small number of non-MHC genes involved in antigen presentation such as CD74 and B2M but its broader genome-wide function and relationship with underlying genetic diversity has not been resolved.

Results: We report the first genome-wide ChIP-seq map for CIITA and complement this by mapping inter-individual variation in CIITA expression as a quantitative trait. We analyse CIITA recruitment for pathophysiologically relevant primary human B cells and monocytes, resting and treated with interferon-gamma, in the context of the epigenomic regulatory landscape and DNA-binding proteins associated with the CIITA enhanceosome including RFX, CREB1/ATF1 and NFY. We confirm recruitment to proximal promoter sequences in MHC class II genes and more distally involving the canonical CIITA enhanceosome. Overall, we map 843 CIITA binding intervals involving 442 genes and find 95\% of intervals are located outside the MHC and 60\% not associated with RFX5 binding. Binding intervals are enriched for genes involved in immune function and infectious disease with novel loci including major histone gene clusters. We resolve differentially expressed genes associated in trans with a CIITA intronic sequence variant, integrate with CIITA recruitment and show how this is mediated by allele-specific recruitment of NF-kB.
\end{abstract}

Conclusions: Our results indicate a broader role for CIITA beyond the MHC involving immune-related genes. We provide new insights into allele-specific regulation of CIITA informative for understanding gene function and disease.

\section{Background}

The transcriptional regulation of the CIITA gene (also referred to as C2TA or MHC2TA) on chromosome $16 \mathrm{p} 13$, and the impact of genetic variation involving this gene for disease, has been extensively characterised following the identification of rare loss-of-function mutations in CIITA resulting in the bare lymphocyte syndrome and severe immunodeficiency due to lack of expression of Major Histocompatibility Complex (MHC) class II genes [1]. CIITA was found to encode a critical non-DNA binding factor, the master MHC class II transactivator, which is

\footnotetext{
* Correspondence: julian@well.ox.ac.uk

${ }^{\dagger}$ Equal contributors

'Wellcome Trust Centre for Human Genetics, University of Oxford, Roosevelt Drive, Oxford OX3 7BN, UK

Full list of author information is available at the end of the article
}

recruited to the class II enhancer complex and plays a critical role in expression of MHC class II genes and, as a result, in the adaptive immune response through antigen presentation to CD4+ T cells [2].

CIITA is expressed in a variety of antigen presenting cells both constitutively and following induction, notably after interferon-gamma (IFNY), with transcriptional regulation of CIITA found to be complex and highly context specific. This includes the occurrence of four different promoters, each with a unique first exon, conferring considerable cellular specificity with, for example, the CIITA class III promoter important for constitutive expression in B cells while the class IV promoter is critical to inducible expression [2-5]. A number of enhancer elements have also been identified, including at least five elements over a $110 \mathrm{~kb}$ region spanning the gene [6]. 
CIITA regulates $\mathrm{MHC}$ class II gene expression through complex mechanisms including chromatin remodelling, transcriptional initiation and elongation [2]. However it does not directly bind DNA. Rather, it is recruited to the proximal promoter regions of the classical MHC class II genes (HLA-DP, HLA-DR and HLA-DQ), and to HLA$D M, H L A-D O$ and the $C D 74$ gene (encoding the molecular chaperone invariant chain which associates with the MHC class II complex) through protein-protein interactions with other components of the MHC class II enhanceosome. These include the regulatory factor $\mathrm{X}$ complex (RFX5, RFXANK and RFXAP), the cAMP responsive element binding protein (CREB1) and activating transcription factor 1 (ATF1), and nuclear factor Y (NFYA/B/C subunits) which bind DNA through the SXY module, a highly constrained series of sequences ( $\mathrm{S}-\mathrm{X}-\mathrm{X} 2-\mathrm{Y}$ boxes) spanning 59 to $68 \mathrm{bp}$ and typically located within $300 \mathrm{bp}$ of the site of transcriptional initiation $[2,7,8]$. In addition, more distal SXY modules with enhancer activity have been identified within the MHC class II region [9]. SXY modules are also found in the MHC class I region where CIITA is recruited but has an ancillary role, the major trans-activator here being NLRC5 [10]. In addition to CD74 and B2M, a small number of non-MHC target genes for CIITA have been identified following chromatin immunoprecipitation (ChIP) for CIITA analysed using promoter arrays [8] and differential gene expression in cell lines deficient in CIITA [11] including RFX5, TPP1, RAB4B, PSMD3 and $M Y B P C 2$ which were found to depend on RFX recruitment to a promoter X-box sequence.

Genome-wide functional genomic approaches provide new opportunities to systematically define such regulatory elements and the impact of genetic variation. Here we describe the first ChIP-seq derived genome-wide map for CIITA occupancy, set in the context of complementary data for other DNA-binding protein members of the CIITA enhanceosome and regulatory features of the epigenomic landscape. We demonstrate how this can be integrated with data mapping the genetic determinants of inter-individual variation in CIITA expression, resolving associated target genes for CIITA both within the MHC and in the larger genomic space outside the MHC. We show how a specific intronic regulatory variant of CIITA is associated in trans with a network of target genes and modulates allele-specific recruitment of NF-KB.

\section{Results}

\section{A genome-wide map of CIITA recruitment in human B cells and monocytes}

In order to generate a high-resolution genome-wide map of CIITA recruitment, we performed ChIP for CIITA followed by high throughput sequencing (ChIP-seq) in primary human B cells and monocytes. Published work to date suggests that CIITA binding is associated with RFX binding to an $\mathrm{X}$ box sequence and our experimental design included generating ChIP-seq data for CIITA and RFX5 using chromatin prepared from the same cells. We analysed CD19+ B cells, which show high constitutive levels of MHC class II expression, together with CD14+ monocytes either resting or treated with IFN $\gamma$ as an inducible paradigm for MHC class II expression, as this cytokine is known to significantly upregulate gene expression [12].

ChIP-seq peaks for CIITA and RFX5 were scored using the model-based analysis of ChIP (MACS) after mapping to the reference human genome haplotype sequence for the MHC region (chr6: 29,580,000-33,100,000). We defined a set of 1,010 binding intervals (BIs) genomewide for CIITA and RFX5 across 12 samples derived from highly purified CD19+ B cells and CD14+ monocyte populations from two different healthy individuals (Figure 1A and Additional file 1: Table S1). Within a given cell type or condition, BIs were defined as CIITA and/or RFX5 enriched ChIP-seq peaks present in both individuals. When we performed an analysis of CIITA and RFX5 affinities (differences in read densities) across the BIs, this partitioned the binding profiles for B cells and monocytes into two distinct groupings consistent with cell type (Additional file 2: Figure S1A). For CIITA, we identified 329 BIs in B cells, 81 in resting monocytes and 591 in monocytes treated with IFN $\gamma$. Specific known and novel CIITA BIs were validated by ChIP for a third individual using a different CIITA antibody (Additional file 2: Figure S2). We integrated all CIITA and RFX5 BIs across the 12 ChIP-seq datasets (Additional file 1: Table S1) with DNase I hypersensitivity site (DHS) maps for primary B cells and monocytes [13]. This revealed that genome-wide, $95 \%$ of CIITA BIs in B cells and $79 \%$ in naïve monocytes are co-incident with a DHS (Additional file 1: Table S1). We investigated the overall relationship with components of the canonical CIITA enhanceosome RFX5, CREB1, ATF1, NFYA and -YB for CIITA BIs. The majority of BIs, $81 \%$ and $98 \%$ for B cells and naïve monocytes, respectively, have data coincidence scores (DCS) greater than 4 (Additional file 1: Table S1 \& Additional file 2: Figure S1B) suggesting that genome-wide, CIITA occupancy involves sites of accessible chromatin and that other constituents of the canonical enhanceosome may be relevant to recruitment.

We proceeded to characterise further the relationship of CIITA and RFX5, defining instances where CIITA BIs are co-incident with RFX5 binding as CIITA Enhanceosome marks (CE-marks) (Figure 1A and $\mathrm{B}$ ). In primary human CD19+ B cells, 329 CIITA and 318 RFX5 BIs were mapped genome-wide (Additional file 1: Table S1) and $69 \%$ of CIITA BIs overlapped with RFX5 to form 


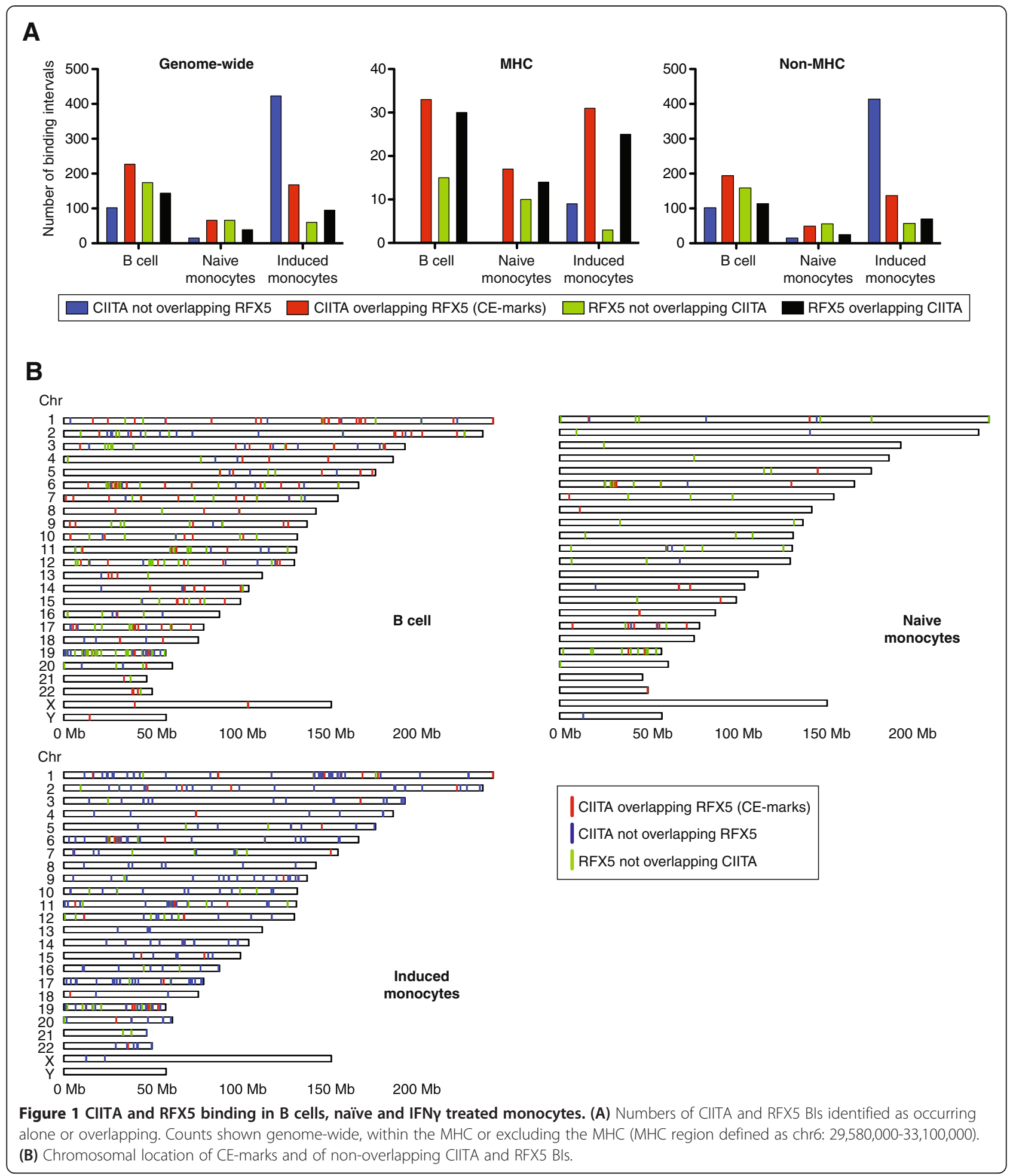

CE-marks (Figure 1A). Within the classical MHC Class II region (chr6: 32,400,000-33,100,000), we found all CIITA BIs were CE-marks. For primary CD14+ monocytes in a naïve state, there were 81 CIITA and 105 RFX5 BIs of which $81 \%$ overlap to form CE marks (Figure 1A) while within the MHC Class II region, there were 17 CIITA BIs that, as observed with B cells, were all CE marks. When monocytes were treated with IFN $\gamma$, we observed 591 CIITA BIs (a 7.3-fold increase compared to the naïve state) with $28 \%$ of CIITA BIs being co-incident with RFX5 and 
forming CE marks. Within the MHC Class II region the proportion of CE marks increased to $78 \%$ and of these, $7 / 31$ were stimulus specific. In contrast, all CIITA BIs within the MHC Class II region of naïve monocytes were also present in treated cells. Similarly, outside of the MHC we observed a lower proportion of CIITA binding in naïve monocytes as compared with induced (Figure 1A). We observed that some CE marks were identified across all cell types and conditions both within and outside of the MHC region. Overall, we find that for $\mathrm{B}$ cells and resting monocytes all CIITA BIs within the MHC class II region were CE marks while only $78 \%$ were CE marks following IFNy treatment of monocytes (Figure 1A). We found that outside the $\mathrm{MHC}$ region, in B cells and naive monocytes $66 \%$ and $77 \%$, respectively, of CIITA BIs were CE marks while after IFN $\gamma$ treatment only $25 \%$ of CIITA BIs in monocytes were identified as CE marks.

We next considered the relationship of CIITA BIs with genes and gene networks. We defined a potential functional relationship with a gene when the distance of a CIITA BI from the transcriptional start site (TSS) of a gene was $<10 \mathrm{~kb}\left(5^{\prime}\right.$ or $\left.3^{\prime}\right)$. Following this definition, we found that 635 of 843 CIITA BIs (75\%) were associated with a gene (Figure 2A), with a total of 442 genes identified (Additional file 1: Table S1). CIITA BIs in B cells, naïve monocytes and monocytes treated with IFN $\gamma$ showed association with 242, 40 and 271 genes, respectively (Additional file 1: Table S1). Across all cell types and conditions, a single BI was found within $2 \mathrm{~kb}$ of the TSS for $75 \%$ to $94 \%$ of these genes. For a further $3 \%$ to $16 \%$ of these genes a single, more distal BI within $10 \mathrm{~kb}$ of the TSS was present. Finally, for the remaining 5\% to 9\% of these genes there was association with both proximal and more distal BIs.

We identified networks enriched for genes associated with a CIITA BI for genome-wide data using DAVID (the database for annotation, visualization and integrated discovery) bioinformatics resources [14,15] and Ingenuity Pathway Analysis tools. The enriched gene networks revealed biological themes related to immunity and inflammation, with antigen presentation the most significantly enriched pathway on global analysis of all CIITA BI associated genes $\left(P=4.7 \times 10^{-19}\right)$ and when CIITA BIs identified in each of the specific cell types were analysed (Additional file 1: Table S1). Signalling involving OX40, a member of the TNFR superfamily important in T cell activation, was also significantly enriched on analysis of all CIITA BI genes $\left(P=1.8 \times 10^{-18}\right)$ and for individual cell types. In terms of disease, we found that autoimmune disease phenotypes were significantly enriched across different cell types in the CIITA BI associated genes. In B cells, RFX5 was the most significant predicted upstream transcriptional regulator $\left(P=1.4 \times 10^{-9}\right)$ together with NLRC5 $\left(P=3.6 \times 10^{-8}\right)$, SRF $\left(P=3.8 \times 10^{-6}\right)$ and
CIITA $\left(P=4.4 \times 10^{-5}\right)$ while in naïve monocytes RFX5 $\left(P=4.6 \times 10^{-9}\right)$ and CIITA $\left(2 \times 10^{-7}\right)$ were most significant (Additional file 1: Table S1). In induced monocytes, a more complex picture was seen with STAT1 $\left(P=9.3 \times 10^{-12}\right)$, NFkB complex $\left(P=1.5 \times 10^{-10}\right)$ and IRF1 $\left(P=1.1 \times 10^{-9}\right)$ identified as upstream regulators together with RFX5 $\left(P=3.3 \times 10^{-9}\right)$.

We proceeded to examine for possible enrichment of genes associated with a CIITA BI among genes that are differentially expressed in monocytes after IFN $\gamma$ treatment [16]. We found significant enrichments of such genes, with highest enrichment when a CE mark was present but there was also an enrichment of genes associated with a CIITA BI where RFX5 was absent (Figure 2B). There was a similar enrichment observed when genes associated with CIITA BIs (combined set of CE marks and CIITA BIs without RFX5) were segregated into known CIITA targets (Additional file 1: Table S1) and novel CIITA targets identified in this study (Figure 2C). At the same time, we also observed that there were other instances whereby differential gene expression between naïve and treated monocytes we previously defined in a large cohort [16] were not associated with a change in CIITA occupancy (Additional file 2: Figure S3).

\section{Resolving proximal and distal sites of CIITA recruitment in the MHC class II region}

CIITA is known to be recruited to the enhanceosome complex at proximal promoter SXY sequences of classical MHC class II genes with a small number of more distal enhancer elements recruiting CIITA identified based on sequence homology with the SXY module [9]. Within the MHC class II region we identified a total of 30 CIITA BIs across freshly isolated B cells and monocytes. The majority (73\%) of these CIITA BIs were located within $10 \mathrm{~kb}$ of the TSS including classical genes such as $H L A-D P, H L A-D R$ and $H L A-D Q$ (Figure 3). Furthermore, for all of these genes there was a CIITA BI located proximally within $2 \mathrm{~kb}$ of the promoter (Additional file 1: Table S1). We observed that IFNy treatment in monocytes resulted in nine additional CIITA BIs not present in naïve cells ( 8 proximal; 1 distal) that were associated with BRD2, PSMB8, HLA-DRA, -DQA, $-D Q B$ and $-D O A$ (Figure 3).

We resolved proximal and distal CIITA recruitment within the MHC class II region as illustrated by the following examples. For $H L A-D R A$, we found evidence of a proximal and a more distal CIITA BI (Figure 4A) with the latter corresponding to a known site for CIITA occupancy [9]. In this instance, both CIITA BIs were associated with RFX5 binding (CE marks), located within transcriptionally accessible regions (DNaseI DHS) and associated with other features of the MHC enhanceosome including binding by CREB1, ATF1, NFYA and -YB. 
A

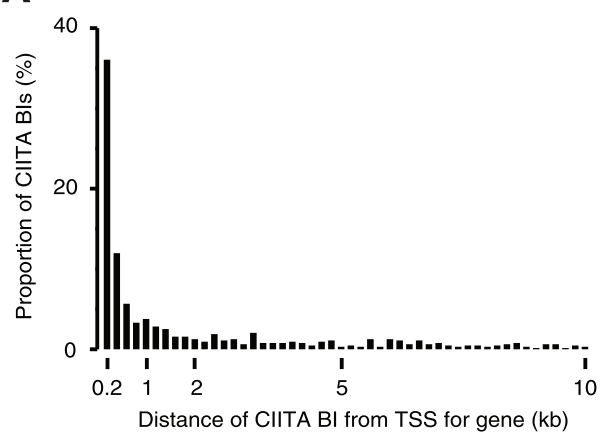

B

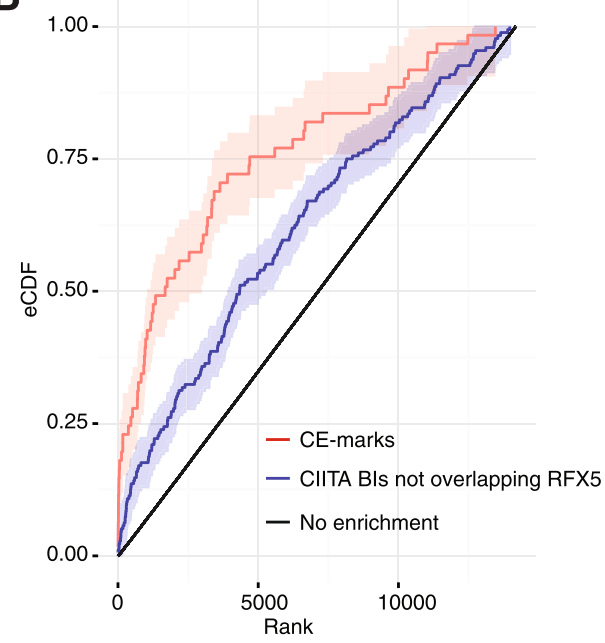

D

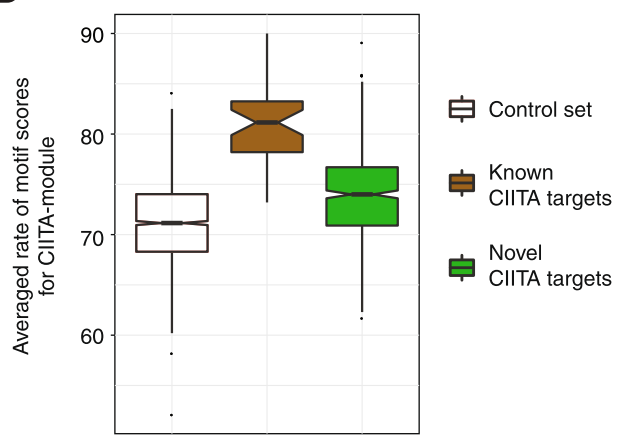

C

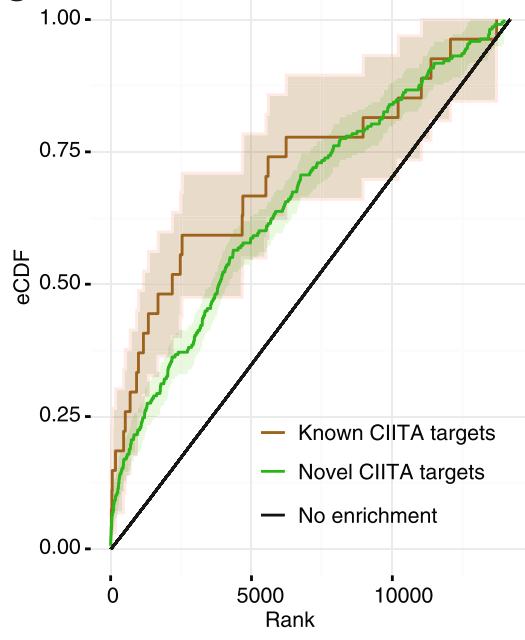

Figure 2 CIITA Bls in relation to gene location, CIITA module and gene function. (A) Distribution of CIITA Bls showing relative distances from the TSS of genes. (B) Enrichment of genes that were differentially expressed in treated monocytes within two groupings of CIITA target genes, one associated with CE marks (CIITA Bls overlapping RFX5) and the other CIITA Bls without RFX5. The empirical cumulative distribution functions (eCDFs) for each grouping is shown with a $95 \%$ confidence interval. A group of CIITA targets containing a larger than expected number of differentially expressed genes will produce an eCDF that lies above the line corresponding to absence of enrichment. (C) Enrichment for all CIITA BIs (overlapping and non-overlapping with RFX5) associated with known and novel CIITA target genes in treated monocytes. (D) Box plot showing averaged rate of MATCH-PWM binding site scores for a CIITA module of RFX5, CREB and NF-Y motifs (co-factors of the classical CIITA enhanceosome) in promoters of known CIITA target genes and those identified in this study (denoted as novel targets where CIITA BIs localised within $10 \mathrm{~kb} 5^{\prime}$ of the TSS). For known and novel target genes, $150 \mathrm{bp}$ intervals centred on CIITA peak summits were analysed. A control set comprised of 1,000 randomly selected segments is included for comparison. Distributions of the three sets were compared using Mann-Whitney-Wilcoxon test, showing that these are significantly different, between the control set and known CIITA targets $\left(P=2.2 \times 10^{-16}\right)$ or novel targets $\left(P=1.5 \times 10^{-11}\right)$, and between known and novel targets $\left(P=2.7 \times 10^{-7}\right)$. 


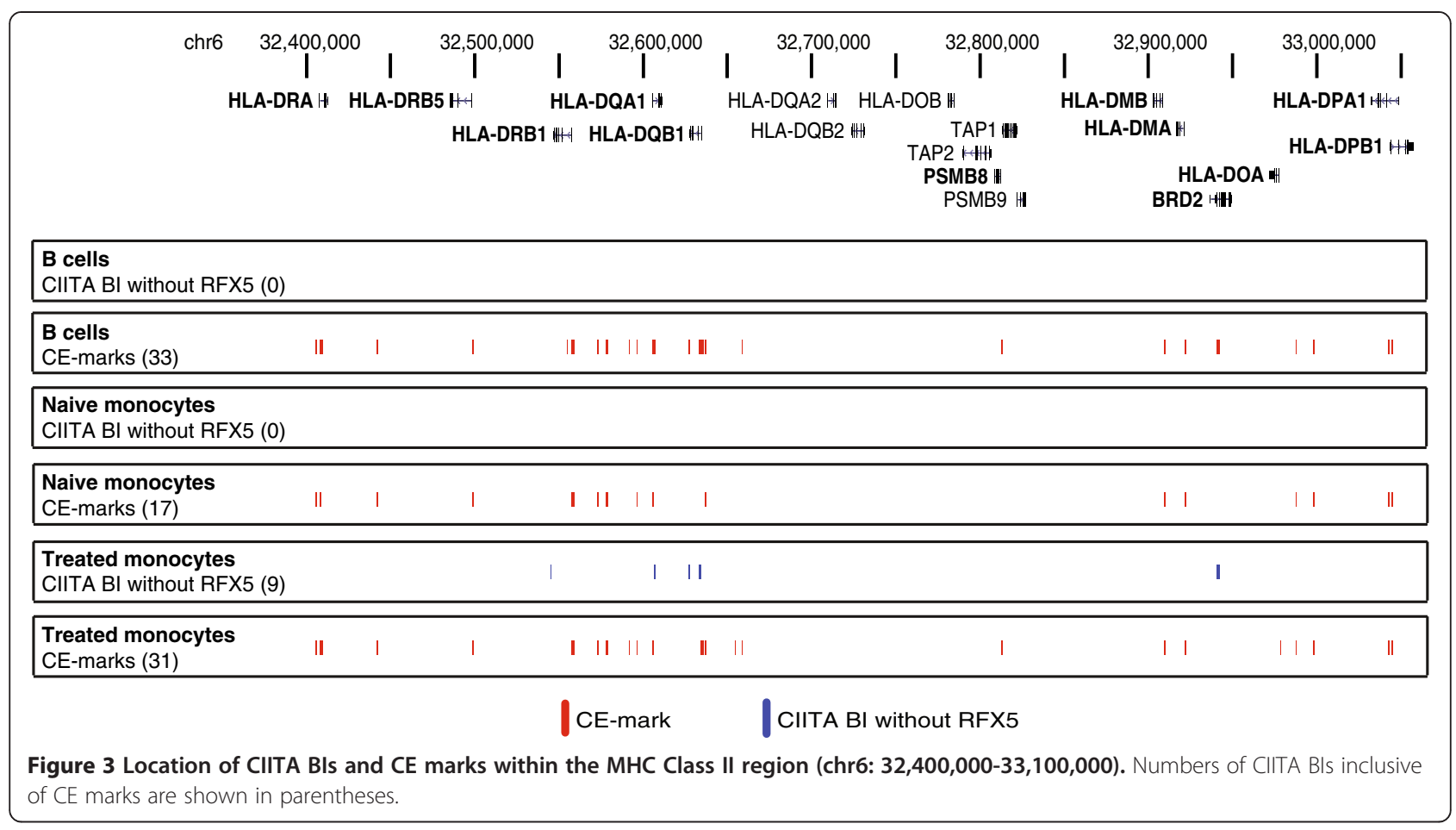

We mapped CIITA recruitment to the proximal promoter regions of $H L A-D R B 1$ and $H L A-D Q A 1$ and identified a further four CIITA BIs between these genes (Additional file 2: Figure S4). These included a CIITA BI localising to an isoform specific promoter for HLA-DQA1 (chr6: 32590730-32591333); a CIITA BI localising to the XL9 intergenic regulatory element previously reported to bind CIITA while also recruiting CTCF to enable chromatin looping and modulation of $H L A-D R B 1$ and $H L A-D Q A 1$ gene expression (chr6: 32595564-32596093) [17]; a previously reported CIITA BI (chr6: 32577249-32578269) [9]; and a novel CIITA BI (chr6: 32571777-32572798) that has not been previously described. All were CE marks in at least a single cell type and condition.

We also identified a distal CIITA BI in an intergenic region $50.9 \mathrm{~kb} \mathrm{5'}$ of HLA-DQA2 (chr6: 3265798632658366 ). This site has previously been reported as a target of CIITA and RFX5 [9] and we found evidence of a CE mark in B cells with inducibility in monocytes treated with IFN $\gamma$. Finally, we resolved in the region spanning HLA-DOA and HLA-DPA1 that there were two proximal promoter CIITA BIs and a further two more distal CIITA BIs (chr6: 32986547-32987178 and chr6: 3299696132997920), both of which were also CE marks. The former is novel while the latter has been reported to be a target of CIITA and RFX5 [9].

Within the MHC class I region (chr6: 2964000031325000), where CIITA plays an ancillary role [10], we identified 11 CIITA BIs of which 10 are CE marks
(Additional file 1: Table S1). These included the proximal promoter regions of classical class I genes including $H L A$ $A, H L A-B, H L A-C, H L A-E$ and $H L A-F$. In addition we found evidence of CE marks at TRIM26 in B cells and monocytes, consistent with earlier reports [8], and novel recruitment at the proximal promoter of PPP1R10 in $\mathrm{B}$ cells (Additional file 2: Figure S5). PPP1R10 encodes the protein phosphatase I regulator PNUTS, recently shown to play a key role in DNA damage repair [18].

\section{Non-MHC CIITA recruitment}

We observed that of 843 CIITA BIs identified across B cells and monocytes, the majority were located outside of the MHC (90\% of CIITA BIs in B cells, $79 \%$ in naïve monocytes, $93 \%$ in treated monocytes). For these CIITA BIs, 40\% were associated with RFX5 binding and classified as CE marks. As observed within the MHC, the majority of CIITA BIs associated with a gene $(<10 \mathrm{~kb}$ from TSS) were similarly localised in terms of proximity to the TSS $(70 \%$ are within $2 \mathrm{~kb}$ of the TSS compared to $82 \%$ within the MHC class II region; Figure $2 \mathrm{~A}$ and Additional file 2: Figure S6) and within transcriptionally accessible regions of the genome (Additional file 2: Figure S1B and Additional file 1: Table S1).

Our global analysis of genes associated with CIITA BIs highlighted gene networks related to immunity and inflammation (Additional file 1: Table S1). Considering all CIITA BIs we identified outside the MHC, we found greater diversity in the associated pathways with EIF2 


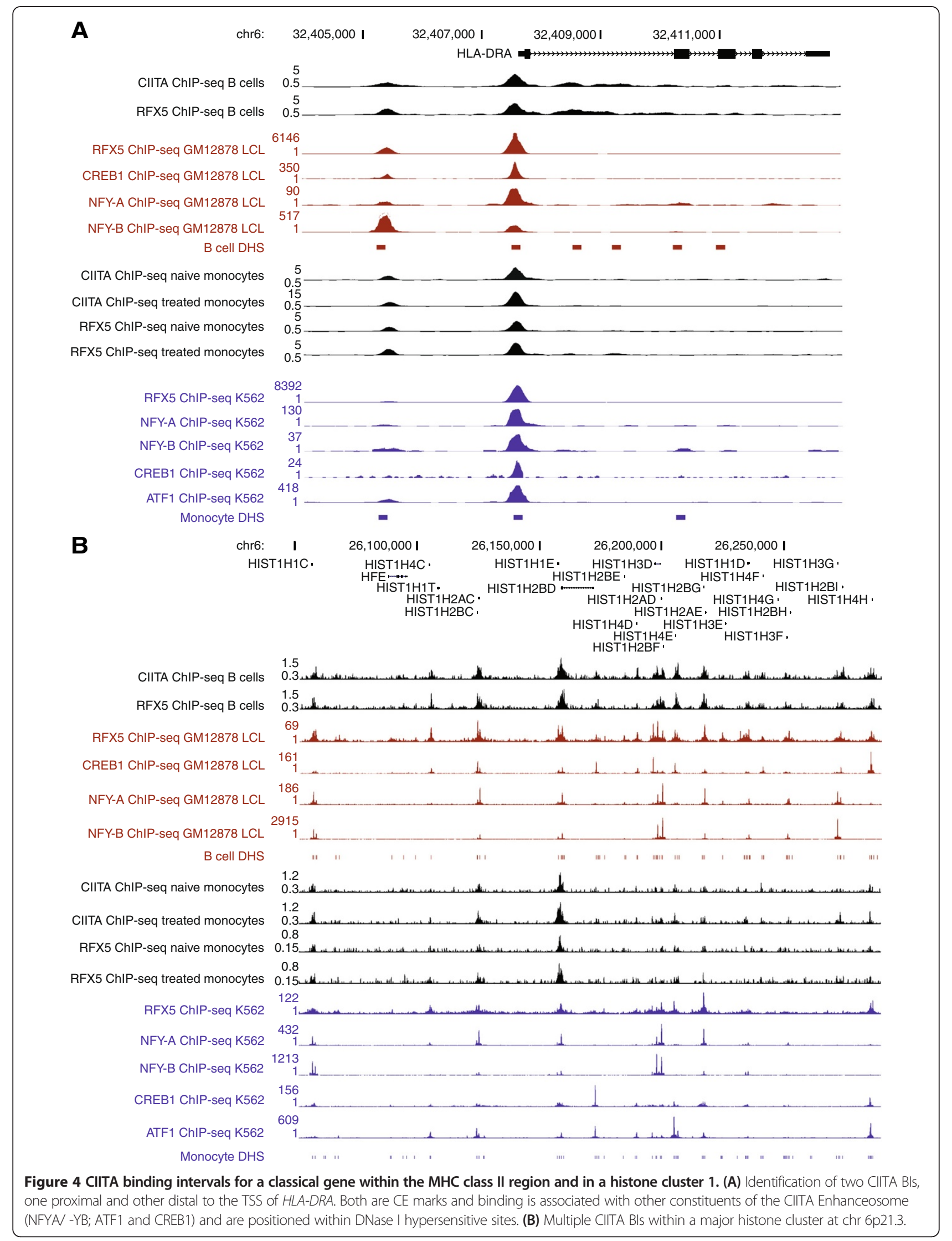


signalling $\left(P=4.5 \times 10^{-10}\right)$ most significant while IL17A and JAK/STAT signalling were also enriched. Cell proliferation $\left(P=7.3 \times 10^{-12}\right)$ and RNA expression $\left(P=4 \times 10^{-11}\right)$ were significantly enriched in terms of biological process. Striking enrichment was also seen for viral infection $\left(P=4.5 \times 10^{-11}\right)$ involving 82 genes together with a number of disease phenotypes involving arthritis (Additional file 1 : Table S1).

CIITA binding has been previously reported for regions outside the $\mathrm{MHC}$, notably $C D 74$ and $B 2 M$ which both encode key components found in MHC class II and class I molecules, respectively $[8,19]$. Our ChIP-seq maps resolved a CE mark located proximally to the TSS of CD74 on chromosome 5 (Additional file 1: Table S1 and Additional file 2: Figure S7). CIITA occupancy at this location has been previously described by Krawczyk and co-workers [8]. There was also a CE-mark located at the proximal $B 2 M$ promoter (Additional file 1: Table S1 and Additional file 2: Figure S8). Our datasets also confirmed previously reported CIITA targets [8] with CIITA BIs including CE marks identified at RFX5, TPP1, $R A B 4 B, Z N F 672, H A U S 5$ and MYBPC2/SPIB. These reported targets had lower $P$ values for the confidence of calling peak summits (Additional file 2: Figure S9).

In both $\mathrm{B}$ cells and treated monocytes, we observed the presence of multiple histone genes within the enriched gene networks (Additional file 1: Table S1). These included the histone $\mathrm{H} 3.3 \mathrm{~B}$ gene $H 3 F 3 B$ for which a CE mark can be found at the proximal promoter (chr17: 73775313-73776112). Within the large histone gene cluster at $6 \mathrm{p} 21.3-6 \mathrm{p} 22$, we identified a further 11 CIITA BIs encompassing four CE marks corresponding to proximal promoter regions of HIST1 genes including HIST1H1C, HIST1H2BC, HIST1H2BD, HIST1H4D, HIST1H2AD, HIST1H4E, HIST1H2BG, HIST1H2BI and HIST1H4H (Figure 4B). We found another grouping of CIITA BIs including CE marks at a second major histone gene cluster with HIST2H2AB and HIST2H2BE at chr1q21.2. In B cells, we identified a CE mark at the proximal promoter of HIST4H4 (Additional file 1: Table S1).

Given that $60 \%$ of CIITA BIs identified outside of the $\mathrm{MHC}$ were not overlapping RFX5 BI, we sought to further characterise CIITA recruitment and possible associated co-factors. We first investigated the occurrence of the tri-motif CIITA module comprised of RFX5, CREB and NF-Y [20] within $150 \mathrm{bp}$ of sequence around peak summits for CIITA BIs localised to within $10 \mathrm{~kb}$ upstream of a TSS. The module was most prevalent for CIITA BIs localised at known CIITA targets that include genes within the MHC Class II region (Figure 2D and Additional file 1: Table S1). The module was also enriched within the novel CIITA BIs identified in this study but significantly less than observed for CIITA BI involving known loci.
We then investigated occurrence of known DNA binding motifs for other transcription factors where CIITA recruitment was occurring in the absence of RFX5 binding. We analysed the 150 bp sequences spanning CIITA peak summits for enriched consensus sequences using the MEME-ChIP suite [21,22]. This showed significant enrichment of several consensus motifs notably STAT3 and SPI1 in IFN-treated monocytes (Additional file 1: Table S1).

To further investigate this, we analysed upstream regulators of genes associated with CIITA BIs not overlapping RFX5 BI using IPA (Additional file 1: Table S1). Analysis of all genes associated with CIITA BIs revealed that while CE marks were most significantly associated with RFX5 $\left(P=4.5 \times 10^{-10}\right)$ and NLRC5 $\left(P=1.4 \times 10^{-8}\right)$, CIITA BI not overlapping RFX5 were significantly enriched for transcription factors STAT1 $\left(P=2.2 \times 10^{-7}\right)$, JUN $\left(P=8.7 \times 10^{-7}\right)$, MYC $\left(P=8.9 \times 10^{-7}\right)$, STAT3 $\left(P=5.4 \times 10^{-6}\right)$ and NFkB complex $\left(7.5 \times 10^{-6}\right)$. A similar pattern was seen when genes associated with CIITA BI in the MHC were compared with non-MHC genes. Considering specific cell types, this effect was most clearly seen on analysis of induced monocytes (Additional file 1: Table S1) with STAT1 $\left(P=2.3 \times 10^{-8}\right)$, NFkB complex $\left(P=2.8 \times 10^{-7}\right)$ and STAT3 $\left(P=3.8 \times 10^{-7}\right)$ the most significantly enriched transcriptional regulators in CIITA BI not overlapping RFX5.

The motif analysis and upstream regulator data raise the hypothesis that members of the STAT family may be involved in CIITA recruitment in the absence of RFX5 but further work is required to test this. In terms of diseases and functions, the overall gene set we identified as associated with CIITA BIs not overlapping RFX5 was most significantly enriched for infectious disease (viral infection) $\left(P=1.6 \times 10^{-8}\right)$ followed by a variety of autoimmune diseases with effects most clearly seen in treated monocytes. In B cells and naïve monocytes enrichment for cancer phenotypes was noted, with death receptor signalling identified on pathway analysis in B cells (Additional file 1: Table S1).

\section{Insights from the genetical genomics of CIITA}

Mapping gene expression as a quantitative trait has the potential to define master regulatory networks through resolution of trans associations in which differential gene expression for a gene set are seen to be associated with a specific genetic marker [23]. We have reported genomewide expression quantitative trait (eQTL) analysis in different primary human cell populations [24]. This global analysis included evidence of a local, cis-eQTL involving CIITA that was specific to B cells. Given the role of CIITA as a master regulator we sought to further resolve this association and to investigate whether there was evidence of associated trans-eQTL networks that could inform CIITA function and modulated genes. 
We used a linear model which incorporated major principal components of the expression data as covariates [25] to further define association within $1 \mathrm{Mb}$ of CIITA for the 281 healthy volunteers using a genomewide panel of 651,210 SNP markers [24]. This resolved that the strongest association involved a single nucleotide polymorphism (SNP) rs11074938 in the 13th intron of CIITA $\left(P=6.9 \times 10^{-20}, \mathrm{FDR}=3.2 \times 10^{-15}\right)$ (Figure 5A to $\left.\mathrm{C}\right)$. Analysis of trans associations involving this SNP (Figure 5D) revealed multiple associations with $\mathrm{MHC}$ Class II genes, notably $H L A-D M A, H L A-D O A$ and $H L A-D R A$ consistent with the known role of CIITA in regulation of these genes. Non-MHC trans associations included genes that are known to show evidence of CIITA recruitment such as RFX5 and TPP1 while for others including OLFML2A and PARVG we find ChIP-seq mapping supports a role for CIITA.

We sought to determine whether the trans association with transcript expression seen for rs11074938 with MHC Class II genes was also observed at the protein level. We assayed MHC class II surface expression on B cells in whole blood from healthy volunteers by multiparametric flow cytometry and observed that individuals possessing one or two copies of the rs11074938 A allele compared to the $\mathrm{G}$ allele expressed higher levels of protein (Figure 5E).

\section{A cis-eQTL for CIITA involving allele-specific recruitment of NF-KB}

In light of the cis and trans associations for rs11074938 in B cells, we sought to investigate the functional basis for this. We first studied the regulatory functional genomic landscape of the sequence variant. Analysis of publically available ENCODE data revealed that SNP rs11074938 was located within a DHS in human primary $B$ cells and lymphoblastoid cell lines (LCLs) but not monocytes (Figure 6A). The position in a region of open chromatin was consistent with the hypothesis that the variant is located in a regulatory element. This was further supported by the presence of specific histone marks including H3K4Me1 and evidence that this is a phylogenically conserved region (vertebrate conserved element, LOD score 31).

Analysis of ChIP-seq data [26] revealed that the SNP was located within an NF-KB target site in LCLs (Figure 6A) and contained an NF-KB consensus binding motif. We investigated whether the A to $G$ substitution could modulate predicted binding affinity using a publically available database identifying binding sites of NF$\mathrm{KB}$ by a combination of protein-binding microarrays and surface plasmon resonance [27]. The ancestral A allele was predicted to show higher binding affinity for NFKB1/ RELA (z-score human 3.25 with A allele vs. 2.83 with G allele) and NFKB2/RELB (z-score 3.16 A vs. <2 G) but the situation is complex with the $\mathrm{G}$ allele also predicted to show higher affinity for the NFKB2 homodimer (z-score $4.38 \mathrm{G}$ vs. $3.86 \mathrm{~A}$ ).

To investigate this further we characterised NF-KB binding by electrophoretic mobility shift assay (EMSA). EMSA using recombinant NFKB1 and RELA proteins demonstrated that with the NFKB1/RELA heterodimer there was greater binding to the $A$ allele compared to the $G$ allele (Figure 6B). Weak binding was observed for NFKB1 alone with no evidence of RELA binding as a homodimer.

We then proceeded to determine whether allelic differences in NF-KB binding were present ex vivo using ChIP-seq data for a panel of five LCLs heterozygous for rs11074938 [26]. This revealed that there was significantly more RELA binding to the A allele compared to the $\mathrm{G}$, with on average $66.1 \%$ (SD 8.1) of reads mapping to the A allele $(P=0.008)$ (Figure $6 \mathrm{C}$ ).

In order to investigate whether the intronic sequence spanning the SNP rs11074938 had regulatory activity that could be modulated in an allele-specific manner, we cloned the region into a reporter gene construct. Given the low transient transfection efficiency of human B cell lines, we performed reporter gene assays in a lymphoblast cell line of $\mathrm{T}$ cell origin (Jurkat). This demonstrated evidence of increased enhancer activity for the A versus the $G$ allele of rs11074938 with allele-specific differences in protein-DNA interactions also seen in this cell line (Additional file 2: Figure S10).

\section{Discussion}

This work defines a high-resolution map of CIITA occupancy within the MHC and genome-wide, presenting evidence for complexity and specificity in the associated enhanceosome complex across different primary peripheral blood cell types and conditions. The results support a role for CIITA beyond the MHC that is often not associated with RFX5 binding but occurs in proximity to genes enriched for immune function and infectious disease, and highlights diverse potential roles including recruitment at histone gene clusters.

The biology of CIITA regulation for MHC class II genes is well established $[2,7,8]$. Our data support evidence for distal as well as classical proximal sites of CIITA recruitment [9], including novel BIs which, as elsewhere in the class II region, appear highly stereotyped in terms of enhanceosome composition and partner DNA binding proteins notably RFX5. Similarly, for the class I region the observed binding is consistent with previous findings at classical genes while supporting evidence for binding sites proximal to TRIM26 and PPP1R10 [10,28].

Genome-wide, the picture is more complex with $60 \%$ of observed CIITA BIs not associated with RFX5 binding based on ChIP-seq performed on the same input chromatin. As seen in the MHC, occupancy was 


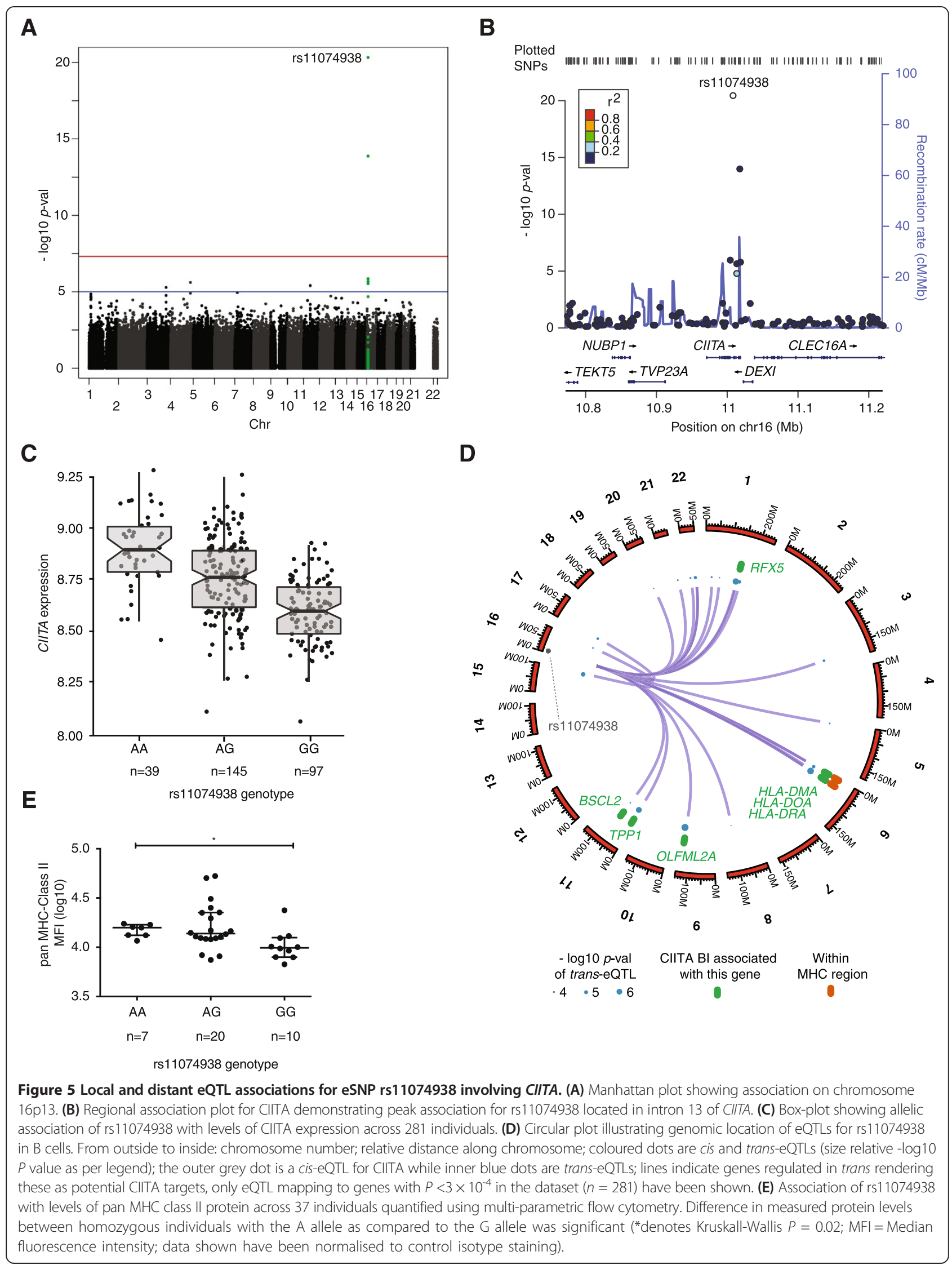




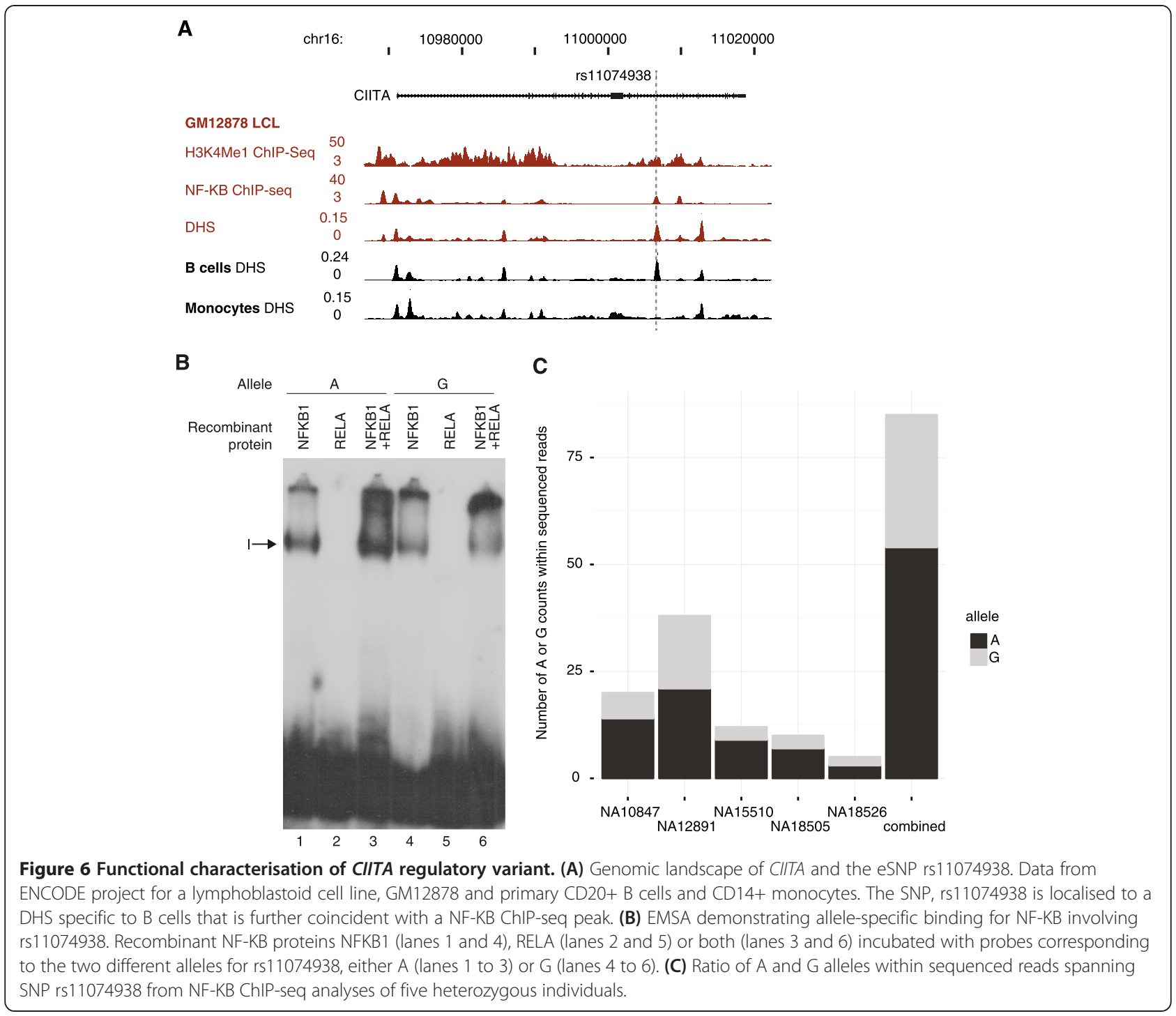

associated with accessible transcriptionally active chromatin and was predominantly within $2 \mathrm{~kb}$ of a gene. The data confirmed some previously identified non-MHC genes such as $C D 74$ and $B 2 M[8,11,19]$ but also highlighted many additional loci notably after monocytes were treated with IFNY in which hundreds of additional BIs were revealed compared to the naïve state consistent with previous observations that CIITA-mediated regulation is intimately linked to treatment with interferons [2]. Examples of genes associated with CIITA BIs include key mediators such as STAT1 and STAT3, observed specifically in monocytes treated with IFNY and B cells, respectively, as well as regions of enrichment for CIITA BIs such as identified within the major histone gene clusters at chromosomes $6 \mathrm{p} 21$ and 1q21.

It has been proposed that in addition to its welldefined enhanceosome function in the MHC, CIITA may function to modulate transcription of a target gene through an alternative mode of action involving formation of a TFIID-like complex in conjunction with partners TAF6, TAF9 and TBP [19]. Within this complex, a TATA-box binding protein (TBP) is proposed to bind DNA and through the involvement of other chromatin modifying enzymes that bring about re-modelling of localised regions within the chromatin, CIITA within this TFIID-like complex may subsequently further dimerise with CIITA in a canonical RFX5-containing enhanceosome. The entire mechanism is however, currently not well understood.

We find that the well characterised, tri-motif CIITA module (RFX5, CREB and NF-Y) was less prevalent within novel CIITA BIs we identified but occurred more frequently than in a control set. Our analysis of peak summits within CIITA BIs not overlapping RFX5 highlighted 
enrichment of the STAT3 consensus DNA binding motif while STAT1 and STAT3 were significant predicted upstream transcriptional regulators for gene sets associated with such CIITA BIs. These data suggest a possible role for STAT family transcription factors in the recruitment of CIITA outside the MHC but further work is required to characterise this.

This work has highlighted the potential informativeness of integrating genomic profiling approaches such as ChIP-seq with trans-eQTL mapping to define modulated gene networks. We identified a strong local association with CIITA expression that we resolved to a specific cis acting expression-associated SNP (eSNP) involving allelespecific recruitment of NF-KB at an intronic regulatory element and differential gene expression. Further work is required to determine the detailed allele-specific transcriptional mechanisms involved in this cis-association. Strikingly, we find this eSNP was associated in trans with a gene network that included classical class II genes demonstrated at the transcript level and subsequently validated at the protein level. Moreover, the trans network also included non-MHC genes, both known targets such as RFX5 and TPP1, and novel genes where we also find evidence of CIITA BIs by ChIP-seq. Our study had limitations in terms of sample size for determining a more extensive modulated gene network and further analysis at different time points following activation and CIITA expression are likely to be required for in-depth profiling and complete integration with maps of CIITA occupancy, together with functional evidence linking recruitment with differences in gene regulation. However the data illustrate that an integrated approach may be highly informative.

\section{Conclusions}

Our study has demonstrated that CIITA recruitment commonly occurs outside the MHC, often not in the setting of a classical enhanceosome with RFX5, and involves genes enriched for immune function and infectious disease. We have functionally characterised a local regulatory variant of CIITA acting through allele-specific recruitment of NFkB which modulates MHC and non-MHC CIITA target genes in trans. The work provides a route-map for further studies to understand the role of CIITA in gene regulation genome-wide, defining complexity and cell type specificity as well as the potential utility of combining analysis of BIs through approaches such as ChIP-seq with experiments of a nature whereby gene networks may be identified through common variants modulating gene expression locally and at a distance through master regulators.

\section{Materials and methods}

Nuclear extracts, electrophoretic mobility shift assays (EMSA) Recombinant NFKB1 (\#31101) and RELA (\#31102) proteins were obtained from Active Motif, UK. Nuclear extracts were prepared from Jurkat cells as previously described [29]. Oligonucleotide probes were radiolabelled with ${ }^{32}$ P-dCTP (Perkin Elmer, Beaconsfield, UK) and EMSA performed as previously described [30]. Probes were generated by annealing forward and reverse oligonucleotides spanning rs11074938 (A_for AGCTGA AAAGGGGAAATGTCTGAAA, A_rev AGCTTTTCA GACATTTCCCCTTTTC; G_for AGCTGAAAAGGG GGAATGTCTGAAA; G_rev AGCTTTTCAGACATT CCCCCTTTTC).

\section{Volunteer recruitment}

The study was approved by the Oxfordshire Research Ethics Committee (COREC reference 06/Q1605/55) and volunteers of European ancestry were recruited in the Oxfordshire area following written informed consent through NIHR Oxford BioBank.

\section{Flow cytometry for analysis of HLA Class II expression}

We assayed HLA class II expression by multi-parametric flow cytometry for whole blood from 37 healthy volunteers using a three-step procedure adapted from a protocol kindly shared by R. Apps from the National Cancer Institute [31]. Human IgG (Sigma-Aldrich, Dorset, UK) was added to each tube, followed by mouse anti-human antibodies directed against all class II alleles (clone Tu39 and SK10/SPV-L3 combined) or an isotype control (murine IgG, Sigma-Aldrich). After $20 \mathrm{~min}$, cells were washed with Dulbecco's phosphate buffered saline (DPBS, Sigma-Aldrich) and sheep IgG was added to each tube followed by sheep-anti-mouse PE (Sigma-Aldrich). After a further 20 -min incubation at $4^{\circ} \mathrm{C}$, cells were washed, secondary antibody sites were blocked with mouse IgG (Sigma-Aldrich) and mouse-anti-human CD19-FITC (clone J3-119, Beckman Coulter) was added. This suspension was incubated for $20 \mathrm{~min}$ at $4^{\circ} \mathrm{C}$, cells were subsequently washed and red blood cells lysed with VersaLyse (Beckman Coulter, High Wycombe, UK) over $20 \mathrm{~min}$, then washed and fixed with Reagent A (Life Technologies, Paisley, UK). Data were acquired immediately on a single FACSCanto (BD) machine using standardized settings verified for each data collection batch using $\mathrm{BD}$ seven-colour setup beads (BD). A minimum of 10,000 gated B cells were acquired for each sample (gating strategy illustrated in Additional file 2: Figure S11). The genotype of samples was anonymised during flow cytometry. Fluorescence-minus-one controls were used to set gate thresholds. Data were analysed in FLowJo (v10.0.6) and Graphpad Prism (v6). Data were normalised for isotype staining by simple subtraction of the isotype MFI in the PE channel from the HLA-II signal.

\section{Isolation of primary immune cells and ChIP-seq}

Leukocyte cones derived from different anonymous individuals were obtained from the NHS Blood and Transplant 
Unit. These were processed using standard techniques as described by Neron and co-workers [32]. Briefly, a Ficoll gradient coupled with successive washes were used to isolate PBMCs from the cones [24]. Automated magnetic activating cell sorting, or autoMACS method (Miltenyi) was used to positively separate CD14+ and CD19+ cell populations starting from 500 million PBMCs following manufacturer's instructions [33]. The technique routinely yields cell purity levels greater than 90\% when cross-validated using a FACS-based assay. CD19+ cells were cross-linked for $10 \mathrm{~min}$ in $1 \%$ formaldehyde, while CD14+ cells were treated with $10 \mathrm{ng} / \mathrm{mL}$ IFNY (R\&D Systems) for $24 \mathrm{~h}$ alongside an untreated control before cross-linkage. Chromatin isolation, sonication using a BioRuptor (Diagenode) and immunoprecipitation using CIITA (Diagenode) and RFX5 (Santa Cruz) ChIP-grade antibodies were performed. Quality control of ChIP-ped DNA was achieved by quantification on a Qubit 2.0 fluorometer (Invitrogen) using the Quant-iT dsDNA HS Assay Kit (Invitrogen). In addition, real-time quantitative PCR was employed to determine enrichment at various genomic locations prior to sequencing (Additional file 2: Supplemental Methods). Approximately 5 to $10 \mathrm{ng}$ of ChIP-ped DNA from each sample was processed into libraries, amplified and sequenced using 51-bp paired-end runs on the Illumina HiSeq (Additional file 2: Supplemental Methods).

\section{ChIP-ddPCR enrichment}

Validation ChIP experiments with two CIITA antibodies (Diagenode C15410062 and SantaCruz sc-48797X) were performed using $\mathrm{B}$ cells and monocytes derived from an independent leukocyte cone. ddPCR was carried out on the QX-100 PCR system (Bio-Rad), using labelled probes and primer sets (sequences listed in Additional file 2: Supplementary Methods) obtained from MetaBion GmbH and following manufacturer's procedures for ddPCR Supermix for Probes (Bio-Rad 186-3010). A duplexed configuration was employed with each well containing an assay for the sample (labelled in FAM) and a second assay for the Rab4A-control region (labelled in YakimaYellow). Enrichment of ChIP over input was determined after normalising for the ratio of corresponding measurements for the control region, Rab4A.

\section{Bioinformatic and statistical analysis including eQTL mapping, peak calling and derivation of Bls}

Stampy version 1.0.21 [34] was employed to align $51 \mathrm{bp}-$ reads to the Human genome UCSC hg19 (GRCh37) using the PGF haplotype as our reference for the MHC region. Processing and filtering of mapped reads were done using Bamtools version 2.3.0 and Samtools version 0.1.17 to remove duplicates, singletons and improperly mapped read pairs that included mappings to non-unique locations and discordant locations. Additionally, read pairs were removed when one or both reads had low mapping quality $(<20)$. Across samples, $38 \%$ to $83 \%$ of read pairs remained after this filtering (Additional file 1: Table S1). Enriched peaks were determined with 'Model-based analysis of ChIP' (MACS version 2.0.10) by comparing ChIP samples to input samples with FDR $<5 \%$. Bedtools version 2.3.0 was used to identify regions shared across the 12 samples. Contiguous, shared regions were merged into a BI when there were enriched peaks for CIITA or RFX5 in both individuals within a single cell type/condition (Additional file 2: Figure S12). This generated 1,010 BIs representative of CIITA and RFX5 binding for further analysis. Plots generated based on BigWig representations of ChIP-seq data in the UCSC Browser show on the y-axis enrichment of peaks (ChIP versus input) with peakenrichment values normalised using the SPMR (signal per million reads) function.

\section{Analysis of known CIITA module (RFX5, CREB and NF-Y) within CIITA BIs}

Three distinct groupings of $150 \mathrm{bp}$ sequence segments around peak summits were analysed: (1) two groups comprised of segments within CIITA BIs that are localised to within $10 \mathrm{~kb}$ upstream or $150 \mathrm{bp}$ downstream of a TSS and that are either known CIITA targets (20 genes) or novel targets identified in this study (275 genes); (2) a control set comprised of 1,000 genes randomly selected from the genome with sequence extracted $10 \mathrm{~kb} 5^{\prime}$ and 150 bp 3' of the TSS from which 150 bp segments randomly selected. The tool Biostrings 2.32.1 from the BioConductor Package was used to scan for presence of RFX5, CREB and NF-Y motifs (source for PWMPosition Weight Matrices used: JASPAR [35], Transfac [36] and Human Protein-DNA Interactome (hPDI) [37]) and score similarity of any motif identified within a segment against the PWM in question. This was carried out iteratively within a segment to obtain a CIITA module with motifs identified in the correct order and with the best score (highest similarity to PWM for all components). An average of scores for the components was computed and this is used as a measure of the prevalence of this known CIITA module within a CIITA BI.

\section{Motif search}

Peak summits in BIs were used to identify enriched consensus sequences with known DNA-binding motifs using MEME-ChIP suite. The peak summits for each sample were extended to $75 \mathrm{bp}$-length sequences into both directions. Equivalent sized DHS in primary cells (ENCODE) were used for background estimation. A customised database containing 569 motifs was created using information from the JASPER and hPDI repositories. We estimated a new Markov model for background 
frequencies. Discovered motifs with similar E-values of less than 1.0 (default option) and $P$ value of less than 0.01 to the known motifs were used for further study. To get motifs similar to known motifs, we searched 'tomtom.txt' output files for each sample and selected motifs occurring in both individuals in the same condition.

\section{Determining biological themes from genes that are regulated by CIITA}

We annotated BIs within $10 \mathrm{~kb}$ from the TSS of the nearest gene using ChIPpeakAnno [38] and CisGenome [39]. Gene clusters formed using CIITA BIs from B cells, naivve and treated monocytes were analysed through the use of the DAVID Bioformatics Suite and Ingenuity Pathway Analysis (IPA, Ingenuity Systems [40]). IPA was used to define gene networks, significant upstream regulators and relationships with canonical signalling pathways.

\section{Enrichment of differentially expressed genes within CE-marks and CIITA BIs}

To investigate whether genes associated with CIITA BIs in treated monocytes are enriched for genes that are differentially expressed when compared to naïve monocytes we used our published gene expression data [16]. Differential gene expression analysis was performed using the limma package. All genes with expression data were ranked by differential expression ( $P$ values). We then considered the distribution of ranks within two different sets of genes; genes associated with a CE mark and genes associated with CIITA BIs without RFX5. We also examined genes associated with CIITA BIs (includes CE marks and CIITA BIs without RFX5) that are either known CIITA targets (Additional file 1: Table S1) or novel targets identified in this study.

\section{Data access}

ChIP-seq datasets have been deposited into the ArrayExpress database [41] under accession number E-MTAB2424.

\section{Additional files}

Additional file 1: Table S1. Worksheets S1.1-1.22.

Additional file 2: Supplemental Methods and Figures S1-12.

\section{Abbreviations}

ATF1: activating transcription factor 1; Bl: binding interval; CE mark: CIITA Enhanceosome mark; ChIP: chromatin immunoprecipitation; ChIP-seq: analysis of ChIP by next generation sequencing; CREB1: CAMP responsive element binding protein; DAVID: database for annotation, visualisation and integrated discovery; EMSA: electrophoretic mobility shift assay; eQTL: expression quantitative trait; eSNP: expression-associated SNP; IFNY: interferon-gamma; LCL: lymphoblastoid cell line; MACS: Model-based analysis of ChIP; MEME: Motif-based sequence analysis tools; MFI: median fluorescence intensity; MHC: Major Histocompatibility Complex; NFY: nuclear factor Y; SNP: single nucleotide polymorphism; SXY: S-XX2-Y boxes; TBP: TATA-box binding protein.

\section{Competing interests}

The authors declare that they have no competing interests.

\section{Authors' contributions}

DW executed ChIP-based experiments. Data from ChIP-seq was analysed by DW, WL and PH. DW and PH contributed to design of the study. SM, EL, VN and KC performed EMSA, flow cytometry, reporter-construct experiments in addition to relevant data analysis. PH and BPF provided analysis and integration of $\mathrm{eQTL}$ data. WL and PH provided all relevant bioinformatics support. KP provided technical support for experiments within the laboratory. DW, WL and JCK drafted the manuscript. JCK conceived, supervised and coordinated the study. All authors have read and approved the manuscript for publication.

\section{Acknowledgements}

We are grateful to all the volunteers who participated in this study and to members of the Knight laboratory for their advice and input. The work was supported by the Wellcome Trust (Grants 074318 (JCK) and 090532/Z/09/Z (core facilities Wellcome Trust Centre for Human Genetics including HighThroughput Genomics Group)), the European Research Council under the European Union's Seventh Framework Programme (FP7/2007-2013)/ERC Grant agreement no. 281824 (JCK), the Medical Research Council (98082 (JCK)) and the NIHR Oxford Biomedical Research Centre.

\section{Author details}

${ }^{1}$ Wellcome Trust Centre for Human Genetics, University of Oxford, Roosevelt Drive, Oxford OX3 7BN, UK. ${ }^{2}$ William Harvey Research Institute, Barts and the London School of Medicine and Dentistry, Queen Mary University London, London, UK.

Received: 24 April 2014 Accepted: 13 October 2014

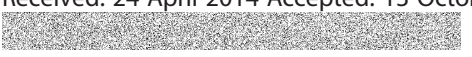

\section{References}

1. Steimle V, Otten LA, Zufferey M, Mach B: Complementation cloning of an MHC class II transactivator mutated in hereditary MHC class II deficiency (or bare lymphocyte syndrome). Cell 1993, 75:135-146.

2. Reith W, LeibundGut-Landmann S, Waldburger JM: Regulation of MHC class II gene expression by the class II transactivator. Nat Rev Immunol 2005, 5:793-806.

3. Lennon AM, Ottone C, Rigaud G, Deaven LL, Longmire J, Fellous M, Bono R, Alcaide-Loridan C: Isolation of a B-cell-specific promoter for the human class II transactivator. Immunogenetics 1997, 45:266-273.

4. Muhlethaler-Mottet A, Otten LA, Steimle V, Mach B: Expression of MHC class II molecules in different cellular and functional compartments is controlled by differential usage of multiple promoters of the transactivator CIITA. EMBO J 1997, 16:2851-2860.

5. Piskurich JF, Gilbert CA, Ashley BD, Zhao M, Chen H, Wu J, Bolick SC, Wright KL: Expression of the MHC class II transactivator (CIITA) type IV promoter in B lymphocytes and regulation by IFN-gamma. Mol Immunol 2006, 43:519-528.

6. Ni Z, Abou El Hassan M, Xu Z, Yu T, Bremner R: The chromatin-remodeling enzyme BRG1 coordinates CIITA induction through many interdependent distal enhancers. Nat Immunol 2008, 9:785-793.

7. Benoist C, Mathis D: Regulation of major histocompatibility complex class-II genes: X, Y and other letters of the alphabet. Annu Rev Immunol 1990, 8:681-715

8. Krawczyk M, Seguin-Estevez Q, Leimgruber E, Sperisen P, Schmid C, Bucher P, Reith W: Identification of CIITA regulated genetic module dedicated for antigen presentation. PLoS Genet 2008, 4:e1000058.

9. Krawczyk M, Peyraud N, Rybtsova N, Masternak K, Bucher P, Barras E, Reith W: Long distance control of MHC class II expression by multiple distal enhancers regulated by regulatory factor $\mathrm{X}$ complex and CIITA. J Immunol 2004, 173:6200-6210.

10. Kobayashi KS, van den Elsen PJ: NLRC5: a key regulator of MHC class I-dependent immune responses. Nat Rev Immunol 2012, 12:813-820.

11. Nagarajan UM, Bushey A, Boss JM: Modulation of gene expression by the MHC class II transactivator. J Immunol 2002, 169:5078-5088.

12. Cullell-Young M, Barrachina M, Lopez-Lopez C, Gonalons E, Lloberas J, Soler C, Celada A: From transcription to cell surface expression, the induction of MHC class II I-A alpha by interferon-gamma in macrophages is regulated at different levels. Immunogenetics 2001, 53:136-144. 
13. Encode Project Consortium: An integrated encyclopedia of DNA elements in the human genome. Nature 2012, 489:57-74

14. da Huang W, Sherman BT, Lempicki RA: Systematic and integrative analysis of large gene lists using DAVID bioinformatics resources. Nat Protoc 2009, 4:44-57.

15. da Huang W, Sherman BT, Lempicki RA: Bioinformatics enrichment tools: paths toward the comprehensive functional analysis of large gene lists. Nucleic Acids Res 2009, 37:1-13.

16. Fairfax BP, Humburg P, Makino S, Naranbhai V, Wong D, Lau E, Jostins L, Plant K, Andrews R, McGee C, Knight JC: Innate immune activity conditions the effect of regulatory variants upon monocyte gene expression. Science 2014, 343:1246949.

17. Majumder P, Gomez JA, Chadwick BP, Boss JM: The insulator factor CTCF controls MHC class II gene expression and is required for the formation of long-distance chromatin interactions. J Exp Med 2008, 205:785-798.

18. Landsverk HB, Mora-Bermudez F, Landsverk OJ, Hasvold G, Naderi S, Bakke O, Ellenberg J, Collas P, Syljuasen RG, Kuntziger T: The protein phosphatase 1 regulator PNUTS is a new component of the DNA damage response. EMBO Rep 2010, 11:868-875.

19. Devaiah BN, Singer DS: CIITA and its dual roles in MHC gene transcription. Front Immunol 2013, 4:476.

20. Zhu XS, Linhoff MW, Li G, Chin KC, Maity SN, Ting JP: Transcriptional scaffold: CIITA interacts with NF-Y, RFX, and CREB to cause stereospecific regulation of the class II major histocompatibility complex promoter. Mol Cell Biol 2000, 20:6051-6061.

21. Bailey $T L$, Machanick $P$ : Inferring direct DNA binding from ChIP-seq. Nucleic Acids Res 2012, 40:e128.

22. Machanick P, Bailey TL: MEME-ChIP: motif analysis of large DNA datasets. Bioinformatics 2011, 27:1696-1697.

23. Westra HJ, Peters MJ, Esko T, Yaghootkar H, Schurmann C, Kettunen J, Christiansen MW, Fairfax BP, Schramm K, Powell JE, Zhernakova A, Zhernakova DV, Veldink JH, Van den Berg LH, Karjalainen J, Withoff S, Uitterlinden AG, Hofman A, Rivadeneira F, t Hoen PA, Reinmaa E, Fischer K, Nelis M, Milani L, Melzer D, Ferrucci L, Singleton AB, Hernandez DG, Nalls MA Homuth $\mathrm{G}$, et al: Systematic identification of trans eQTLs as putative drivers of known disease associations. Nat Genet 2013, 45:1238-1243.

24. Fairfax BP, Makino S, Radhakrishnan J, Plant K, Leslie S, Dilthey A, Ellis P, Langford C, Vannberg FO, Knight JC: Genetics of gene expression in primary immune cells identifies cell type-specific master regulators and roles of HLA alleles. Nat Genet 2012, 44:502-510.

25. Biswas S, Storey JD, Akey JM: Mapping gene expression quantitative trait loci by singular value decomposition and independent component analysis. BMC Bioinformatics 2008, 9:244.

26. Kasowski M, Grubert F, Heffelfinger C, Hariharan M, Asabere A, Waszak SM, Habegger L, Rozowsky J, Shi M, Urban AE, Hong MY, Karczewski KJ, Huber W, Weissman SM, Gerstein MB, Korbel JO, Snyder M: Variation in transcription factor binding among humans. Science 2010, 328:232-235.

27. Siggers T, Chang AB, Teixeira A, Wong D, Williams KJ, Ahmed B, Ragoussis J, Udalova IA, Smale ST, Bulyk ML: Principles of dimer-specific gene regulation revealed by a comprehensive characterization of NF-kappaB family DNA binding. Nat Immuno/ 2012, 13:95-102.

28. Neefjes JJ, Momburg F, Hammerling GJ: Selective and ATP-dependent translocation of peptides by the MHC-encoded transporter. Science 1993, 261:769-771.

29. Schreiber E, Matthias P, Muller MM, Schaffner W: Rapid detection of octamer binding proteins with 'mini-extracts', prepared from a small number of cells. Nucleic Acids Res 1989, 17:6419.

30. Udalova IA, Knight JC, Vidal V, Nedospasov SA, Kwiatkowski D: Complex NF-kappaB interactions at the distal tumor necrosis factor promoter region in human monocytes. J Biol Chem 1998, 273:21178-21186.

31. Thomas R, Thio CL, Apps R, Qi Y, Gao X, Marti D, Stein JL, Soderberg KA, Moody MA, Goedert JJ, Kirk GD, Hoots WK, Wolinsky S, Carrington M: A novel variant marking HLA-DP expression levels predicts recovery from hepatitis B virus infection. J Virol 2012, 86:6979-6985.

32. Neron S, Thibault L, Dussault N, Cote G, Ducas E, Pineault N, Roy A: Characterization of mononuclear cells remaining in the leukoreduction system chambers of apheresis instruments after routine platelet collection: a new source of viable human blood cells. Transfusion 2007, 47:1042-1049.

33. Willasch A, Eing S, Weber G, Kuci S, Schneider G, Soerensen J, Jarisch A, Rettinger $\mathrm{E}$, Koehl U, Klingebiel T, Kreyenberg H, Bader P: Enrichment of cell subpopulations applying automated MACS technique: purity, recovery and applicability for PCR-based chimerism analysis. Bone Marrow Transplant 2010, 45:181-189.

34. Lunter G, Goodson M: Stampy: A statistical algorithm for sensitive and fast mapping of Illumina sequence reads. Genome Res 2010, 21:936-939.

35. Mathelier A, Zhao X, Zhang AW, Parcy F, Worsley-Hunt R, Arenillas DJ, Buchman S, Chen CY, Chou A, lenasescu H, Lim J, Shyr C, Tan G, Zhou M, Lenhard B, Sandelin A, Wasserman WW: JASPAR 2014: an extensively expanded and updated open-access database of transcription factor binding profiles. Nucleic Acids Res 2014, 42:D142-D147.

36. Matys V, Kel-Margoulis OV, Fricke E, Liebich I, Land S, Barre-Dirrie A, Reuter I, Chekmenev D, Krull M, Hornischer K, Voss N, Stegmaier P, Lewicki-Potapov B, Saxel $\mathrm{H}$, Kel AE, Wingender E: TRANSFAC and its module TRANSCompel: transcriptional gene regulation in eukaryotes. Nucleic Acids Res 2006, 34:D108-D110.

37. Xie Z, Hu S, Blackshaw S, Zhu H, Qian J: hPDI: a database of experimental human protein-DNA interactions. Bioinformatics 2010, 26:287-289.

38. Zhu LJ, Gazin C, Lawson ND, Pages H, Lin SM, Lapointe DS, Green MR: ChIPpeakAnno: a Bioconductor package to annotate ChIP-seq and ChIP-chip data. BMC Bioinformatics 2010, 11:237.

39. Ji H, Jiang H, Ma W, Johnson DS, Myers RM, Wong WH: An integrated software system for analyzing ChIP-chip and ChIP-seq data. Nat Biotechnol 2008, 26:1293-1300.

40. Qiagen: QIAGEN's Ingenuity Pathway Analysis. IPA ${ }^{\oplus}$, QIAGEN Redwood City. [www.qiagen.com/ingenuity]

41. Rustici G, Kolesnikov N, Brandizi M, Burdett T, Dylag M, Emam I, Farne A

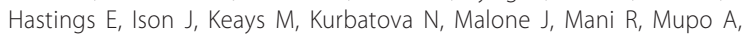
Pedro Pereira R, Pilicheva E, Rung J, Sharma A, Tang YA, Ternent T, Tikhonov A, Welter D, Williams E, Brazma A, Parkinson H, Sarkans U: ArrayExpress update-trends in database growth and links to data analysis tools. Nucleic Acids Res 2013, 41:D987-D990.

\section{doi:10.1186/s13059-014-0494-z}

Cite this article as: Wong et al:: Genomic mapping of the MHC transactivator CIITA using an integrated ChIP-seq and genetical genomics approach. Genome Biology 2014 15:494.

\section{Submit your next manuscript to BioMed Central and take full advantage of:}

- Convenient online submission

- Thorough peer review

- No space constraints or color figure charges

- Immediate publication on acceptance

- Inclusion in PubMed, CAS, Scopus and Google Scholar

- Research which is freely available for redistribution 